\title{
Zu Theodoros Pediasimos. ${ }^{1}$ )
}

In dem wertvollen Beitrage zur Litteratur des 14. Jahrh., dessen Titel unten angeführt ist, hat mein Freund M. Treu mehrere Texte herausgegeben und dieselben nicht nur mit dem nötigen kritischen Apparate, sondern auch mit nützlichen, historischen und anderen, Anmerkungen versehen. Die Texte sind in mehrfacher Hinsicht interessant, manche von denselben stehen sogar geschichtlich und topographisch in naher Verbindung mit meiner, vom Herausgeber benutzten ${ }^{2}$ ),

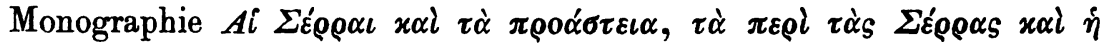

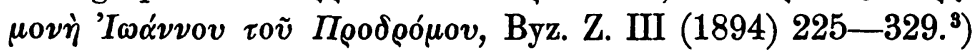

1) Von Theodoros Pediasimos, welcher in den ersten Dezennien des 14. Jahrhunderts lebte, in Thessalonike studierte und in Serrai, wenn nicht geboren wurde, doch längere Zeit wohnte, liegen vor:

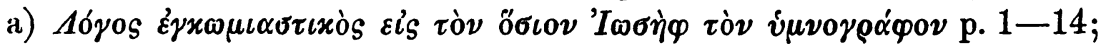

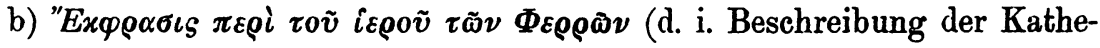

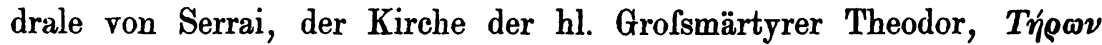

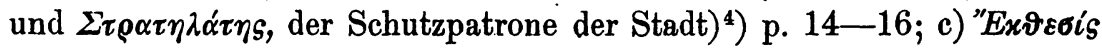

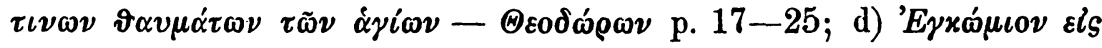

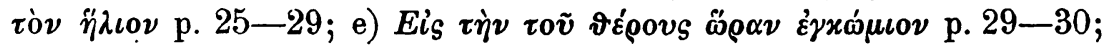

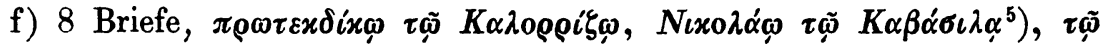

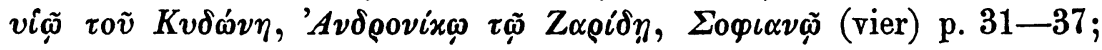

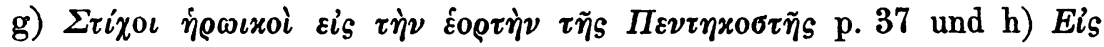

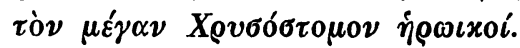

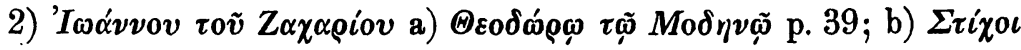

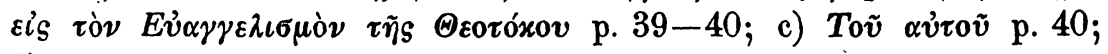

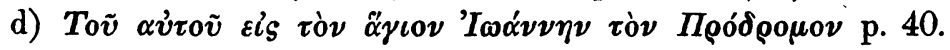

1) M. Treu, Theodori Pediasimi eiusque amicorum quae exstant, Potsdam 1899. Griechischer Text (p. 1-49) mit lateinischem Kommentare (p. 50-61).

2) Cf. Krumbacher, B. Litt. ${ }^{2}$ S. 486-487.

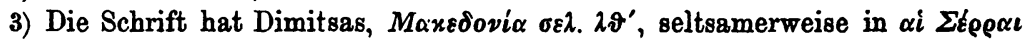
$x \alpha i \tau \dot{\alpha} \pi \varepsilon \rho i \chi \omega \rho \alpha$ umgetauft!

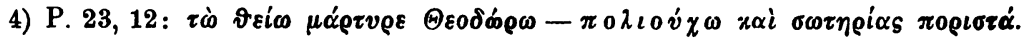

5) Das Epitheton hört man noch jetzt in Didymoteichon und vielleicht such anderswo. 


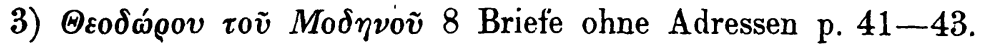

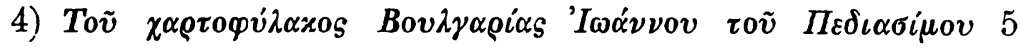

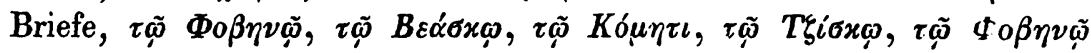
p. $44-48$.

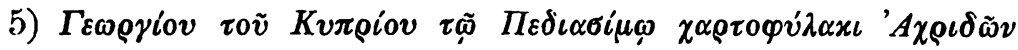
p. $48-49$.

A) Die Stadt von Serrai nennen Th. Pediasimos und seine Freunde nach der byzantinischen Gewohnheit (Treu p. 53; Papag., Byz. Z. III

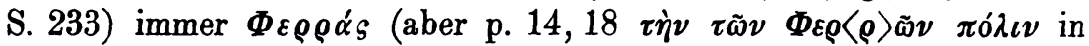
marg. rubro $\gamma \rho \tau \tilde{\omega} \nu \sigma \varepsilon \rho\langle\rho\rangle \tilde{\omega} \nu$ und p. 18, $12 \varepsilon \varepsilon^{\prime} \sigma \omega \Phi_{\varepsilon \varrho \rho \tilde{\omega} \nu}$ in marg. 2 man. $\sigma \varepsilon \oint \rho \tilde{\omega} \nu)$. Den von Treu (p. 54) zuerst bekannt gemachten Namen $\Sigma \varphi \varepsilon ́ \rho \rho \alpha s$ (in einem Briefe von Konst. Akropolitis in cod. Ambrosiano Gr. II 81 sup.) kann man wohl für ein Gemisch von

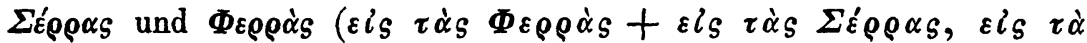
$\left.\Sigma \varphi \varepsilon^{\prime} \rho \alpha_{S}\right)$ ansehen; cf. B. Z. S. $316 \varepsilon i s \tau \dot{\alpha} s " E \rho \alpha S=\varepsilon i_{S} \tau \dot{\alpha} \Sigma \varepsilon^{\prime} \rho \rho \alpha s$, worüber B. Z. S. 234.

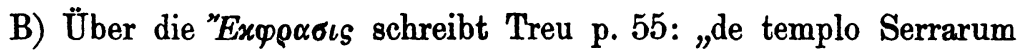
primo - confer Petri Papageorgiu dissertationem -. Quae hic de templo proposuit Pediasimus vereor ne eorum qui harum rerum periti sunt exspectationem decipiant" etc. Vengleichen lassen sich p. 14, 28:

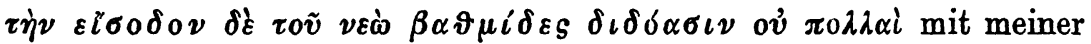

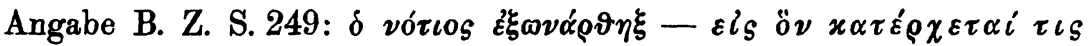
$\delta \iota \dot{\alpha} \delta$ v́o $x \lambda \iota \mu \alpha \dot{x} \omega \nu \dot{\varepsilon} x \pi \varepsilon \nu \tau \varepsilon x \alpha i \delta \varepsilon x \alpha \beta \alpha \vartheta \mu i \delta \omega \nu ;$ p. 14, 30: $\dot{\varepsilon} \pi \alpha \nu$ -

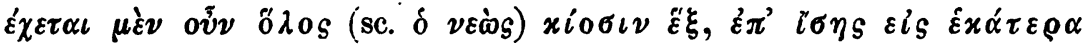

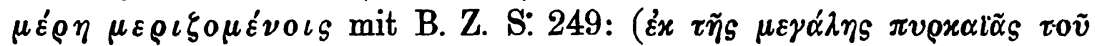

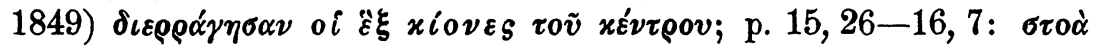

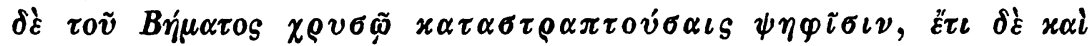

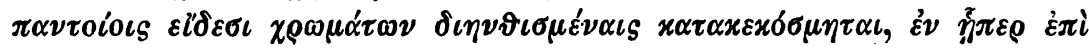

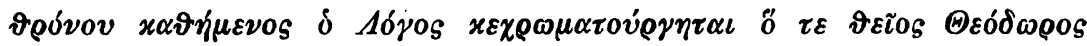

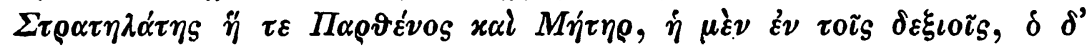

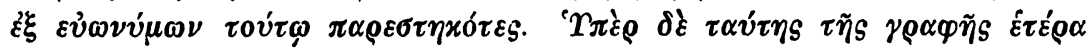

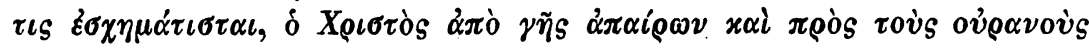

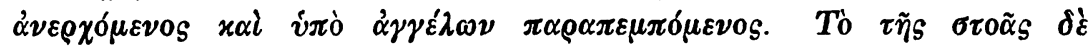

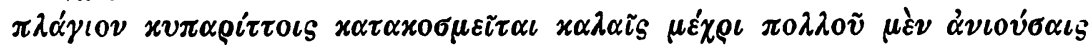

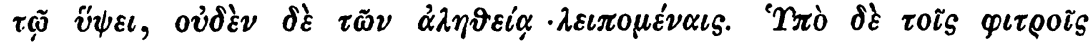

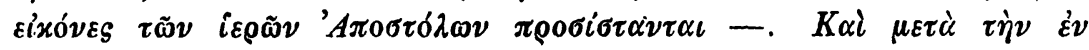

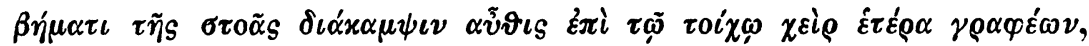

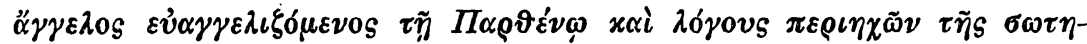

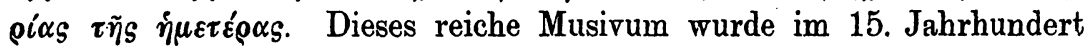
durch ein anderes ersetzt, B. Z. S. 249-250: To "A $A \iota \nu v B \tilde{\eta} \mu \alpha-\varepsilon^{\prime \prime} \chi \varepsilon \iota$

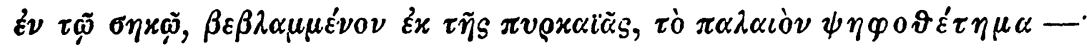




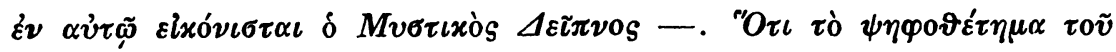

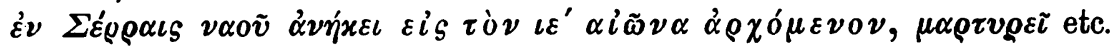

C) Auch in der Explanatio miraculorum finden sich manche interessante Andeutungen über die Stadt und die Umgebung. P. 20, 10:

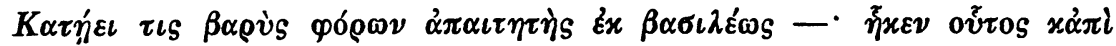

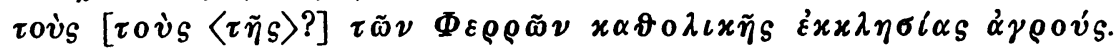

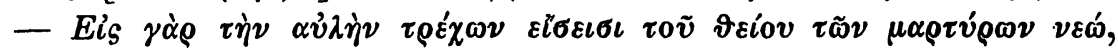

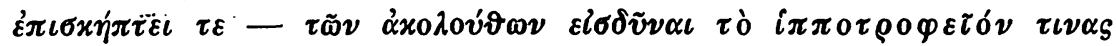

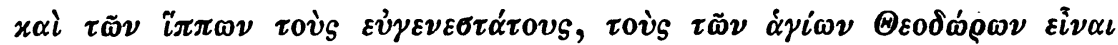

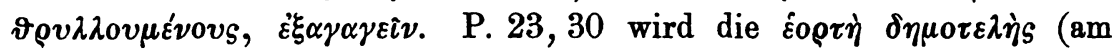

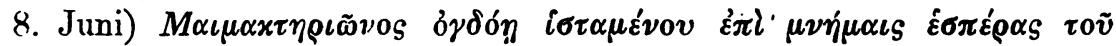

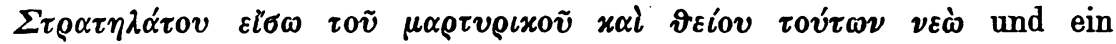
Wunder der Hll. beschrieben (Treu p. 55); B. Z. S. 277 in den $\Sigma$ tixoı $\varepsilon i S \Sigma \tau \rho \alpha \tau \eta \lambda \alpha^{\prime} \tau \eta \nu$ des Metropoliten von Serrai Gennadios: ő $\lambda\llcorner\iota \nu \delta \gamma \delta$ ó $\tau \eta$

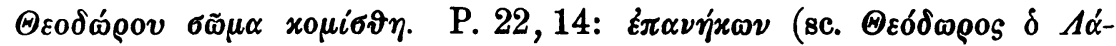

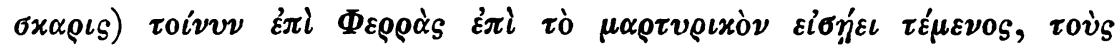

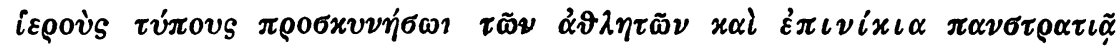

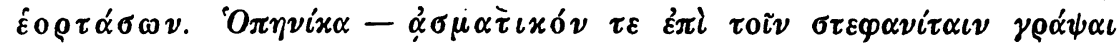

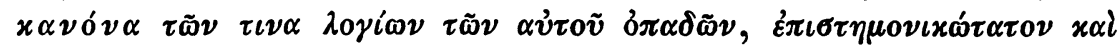

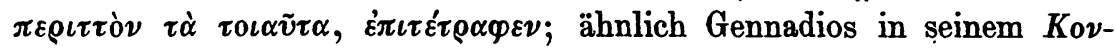
$\tau \alpha^{\prime} x \iota \nu \nu$ (B. Z. S. 277): $\Sigma \varepsilon \rho \rho \tilde{\omega} \nu \pi \rho \circ \mu \alpha ́ \chi 0 \iota \nu \pi \rho \circ \sigma \alpha v \delta \tilde{\omega} \quad \tau \dot{\alpha} . \quad \chi \alpha \rho \iota-$

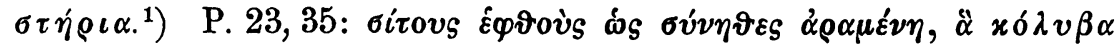

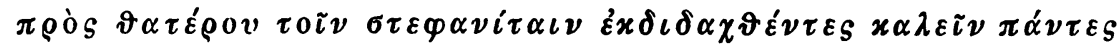

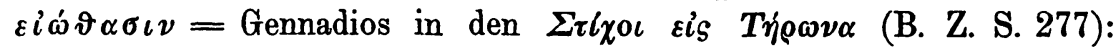

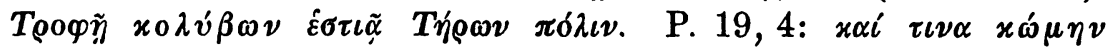

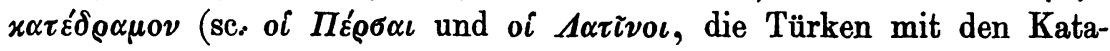

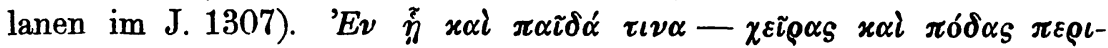

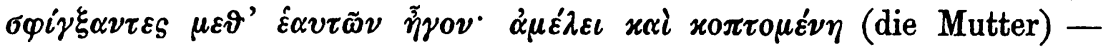

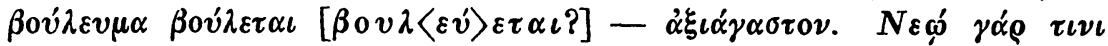

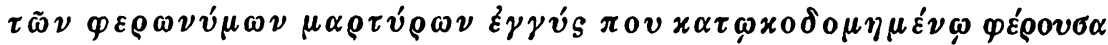

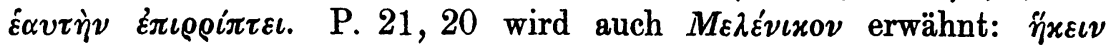

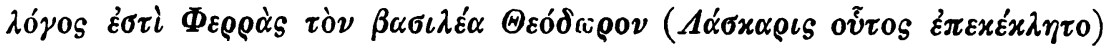

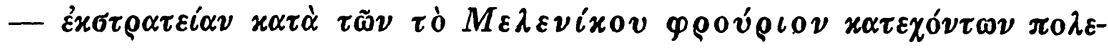

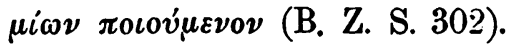

D) Zum Briefe an den Protekdikos Kalorrizos schreibt Treu p. 55: „Petrus Papageorgiu BZ - primus edidit tabulas, quibus Hypomone monacha anno 1344 monasterium S. Georgii Cryoneritae ante Serras situm monasterio S. Iohannis Praecursoris et Baptistae donat. has

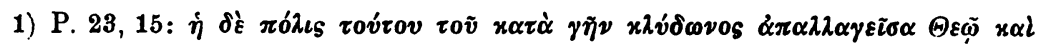

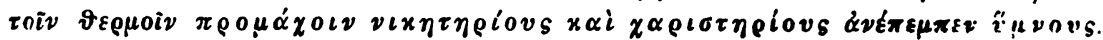




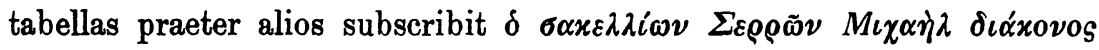
$\delta K \alpha \lambda o ́ \rho \varrho \iota \zeta o$ s."

E) Über das Geschlecht von Theodoros Modinos handelt Treu p. 60: „Theodori Modeni gentem Serrensem esse etiam aliunde scimus.

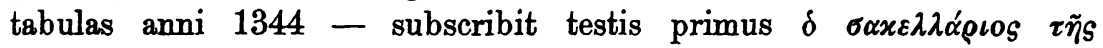
$\dot{\alpha} \gamma \iota \omega \tau \alpha \dot{\tau} \tau\}$ tabulis ipsis ab Hypomone commemoratur $\dot{\eta} \vartheta v \gamma \alpha^{\prime} \tau \eta \rho \mu v$ i $\dot{\varepsilon}^{\prime} \nu \tau \iota \mu \rho \tau \alpha^{\prime} \tau \eta$ $\Sigma \alpha x \varepsilon \lambda \lambda \alpha \rho \varepsilon^{\prime} \alpha$ [so in der Urkunde] $\dot{\eta} \operatorname{Mo\delta } \eta v \eta^{\prime} . "$

F) Das dritte Gedichtchen von Johannes Zacharias $\varepsilon i s$ rò $\nu$ ä $\gamma \iota \nu \nu$

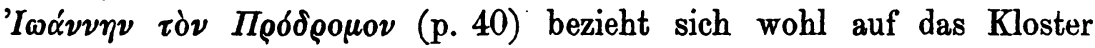
des Prodromos:

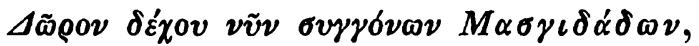

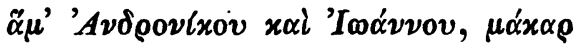

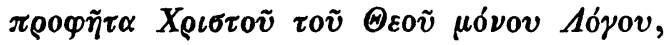

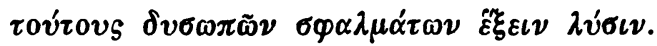

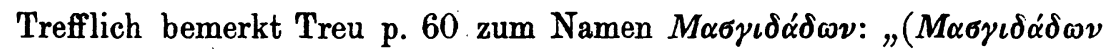
nomen nondum legi:) derivatum est a verbo $\mu \alpha \sigma \gamma i \delta \iota \nu \nu$, quod Turcice est

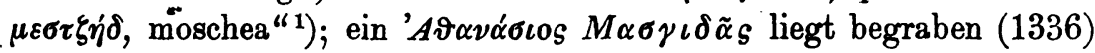
im Kloster von Prodromos, wie folgende Inschrift beweist ${ }^{2}$ ):

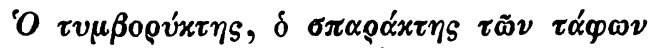

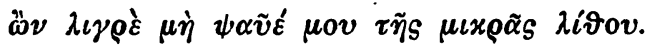

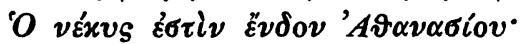

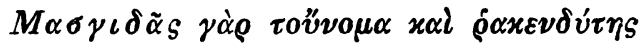

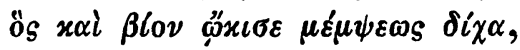

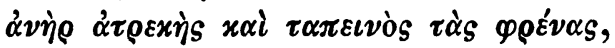

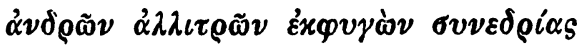

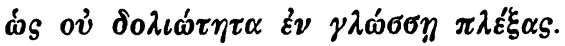

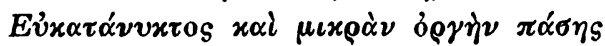

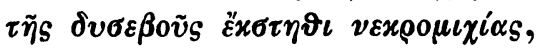

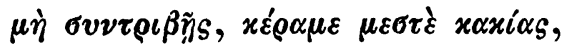

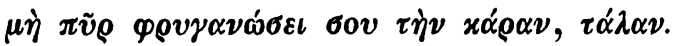

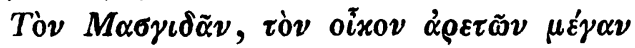

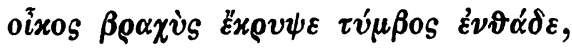

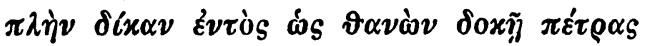

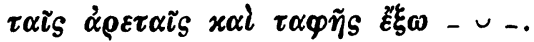

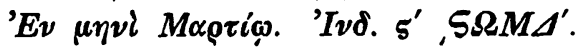

1) Ngr.-barb. $\mu \varepsilon \chi \tau \sigma i \tau \iota(o v)$, portug. Mesquita.

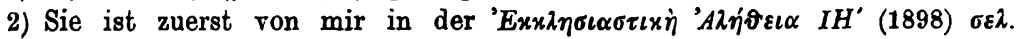
442-443 nach einer Kopie des jetzigen Higumenos Christophoros, B. Z. III S. 317 (in kleinen Buchstaben) herausgegeben. 
Den Stein sah ich im J. 1890 im Exonarthex der Kirche liegen, hatte aber keine Zeit, das Epigramm abzuschreiben. Des Steinmetzen

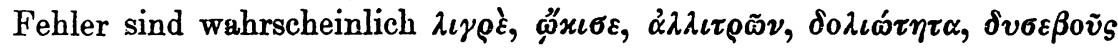
und $\varphi \varrho v \gamma \alpha \nu \omega ́ \sigma \varepsilon \iota$ (st. $\varphi \rho v \gamma \alpha \nu \omega ́ \sigma \eta)$. Dagegen sind ( $\mu \iota x \rho \dot{\alpha} \nu) \delta \varrho \gamma \dot{\nu} \nu$

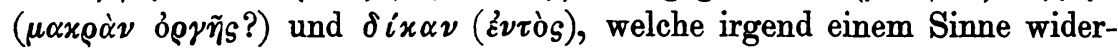
stehen, einem Mifsverständnisse der bezüglichen Kompendien zuzuschreiben. Der Sinn der V. 15-16 scheint mir zu sein: $\pi \lambda \dot{\nu} \nu$ மs

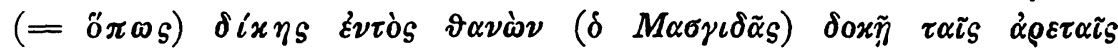

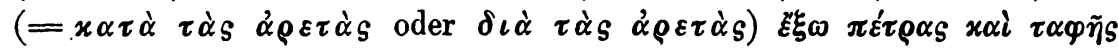

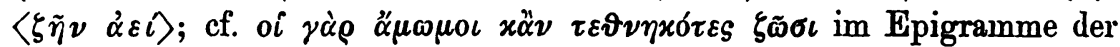
hl. Theodora, welches ich B.Z. X 149 mitgeteilt habe. Schön ist der Gegen-

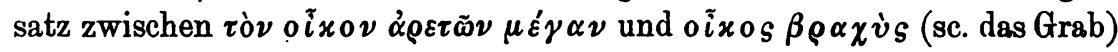

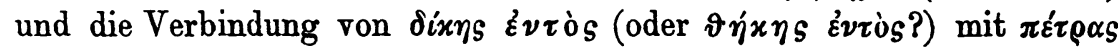
$\ddot{\varepsilon} \xi \omega$. Statt $\nu \varepsilon x \rho \circ \mu \iota \chi i \alpha s$ stand wohl $\nu \varepsilon x \rho о \mu о \iota \chi \varepsilon i \alpha S$ oder vielmehr

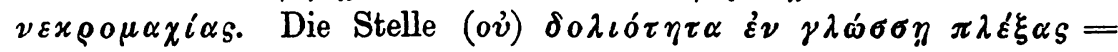

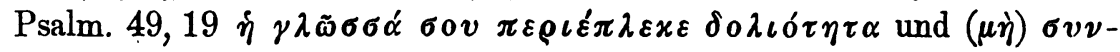

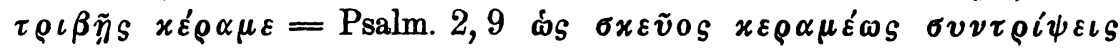

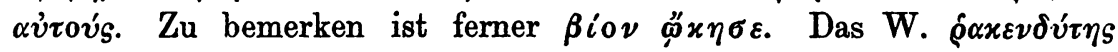
beweist, dafs Athanasios ein Mönch war, höchstwahrscheinlich Bruder von Andronikos und Johannes $\tau \tilde{\omega} \nu$ M $\alpha \sigma \gamma \iota \delta \alpha \delta \omega \nu$, im Auftrage deren

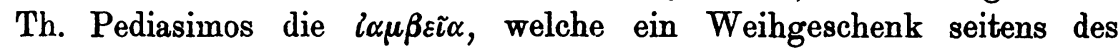
Bruderpaares begleiten sollten, verfalste. Die W. $\tau v \mu \beta o \rho v ́ x \tau \eta s, \nu \varepsilon-$ $x \rho \circ \mu \alpha \chi \ell \alpha$ und $\varphi \varrho v \gamma \alpha \nu \tilde{\omega}$ fehlen selbst bei Sophocles, Gr. Lexicon.

Folgende Vermutungen zum Texte empfehle ich dem verdienstrollen Herausgeber zu gefälliger Berücksichtigung.

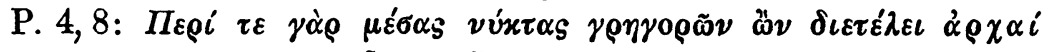

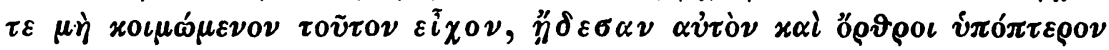
оі́

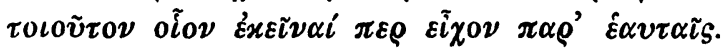

Treu schlug $\alpha \varrho \alpha i$ vor; wenn zu $\alpha \varrho \chi \alpha i$ nicht $\nu v x \tau \tilde{\omega} \nu$ hinzuzudenken

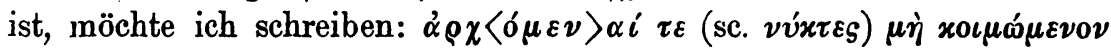

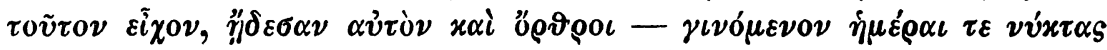

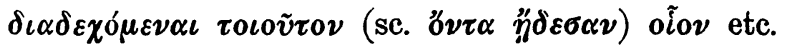

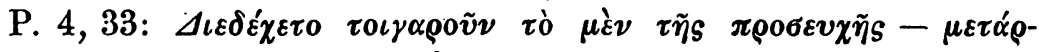

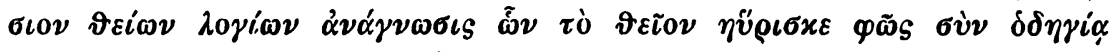

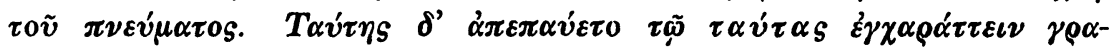

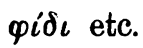

$T \alpha \tilde{v} \tau \alpha$ ? (sc. $\tau \dot{\alpha} \vartheta \varepsilon \tilde{\alpha} \alpha \lambda \hat{\gamma} \iota \alpha)$.

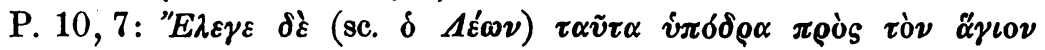

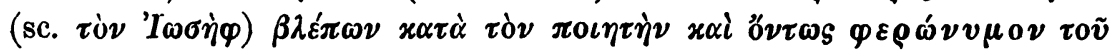

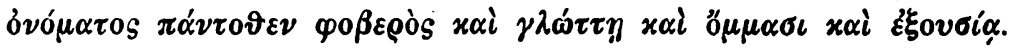


Ist $\varphi \varepsilon \rho \omega ́ \nu v \mu o \nu$ nicht ein Adv. (= $\left.=\varepsilon \rho \omega \nu v^{\prime} \mu \omega s\right)$, so schlage jch $\varphi \varepsilon \rho \omega ́ \nu v \mu o s$ vor (ein Wortspiel mit $\Lambda \varepsilon \dot{\omega} v$ ).

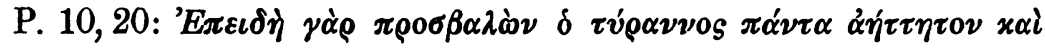

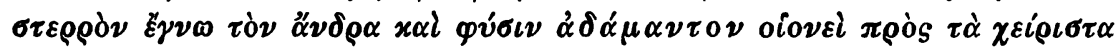

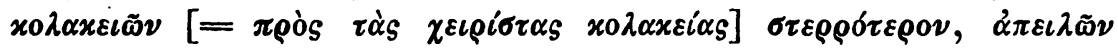

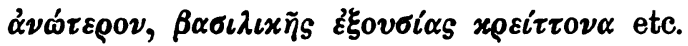

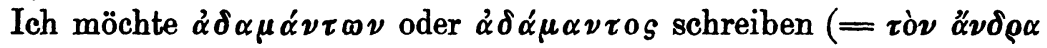

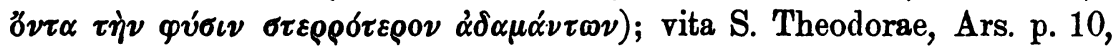

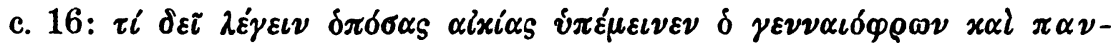

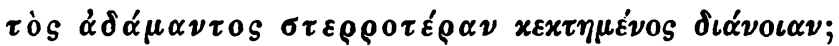

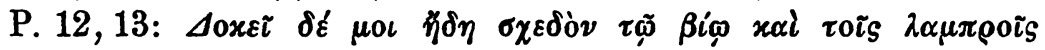

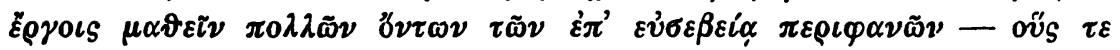

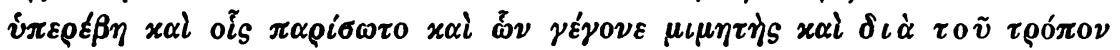
$\tau i \nu$ '

$\Delta \iota \grave{\alpha} \tau 0 \tilde{v}\langle\tau 0\rangle \tau \varrho o ́ \pi 0 \nu$ ?

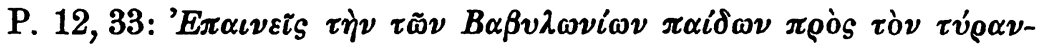

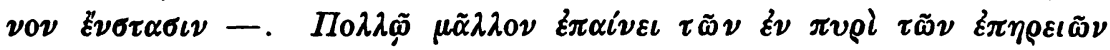

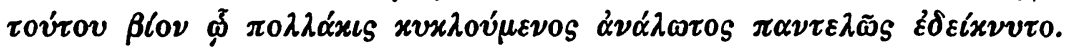

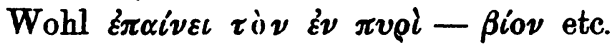

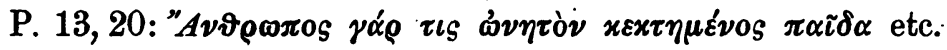
$\mu \varepsilon \dot{\nu} 0 \nu$.

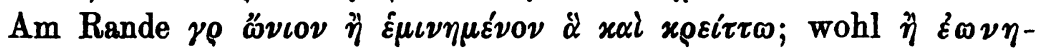

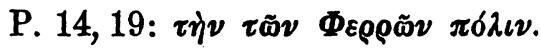

Die Hs

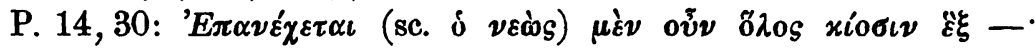

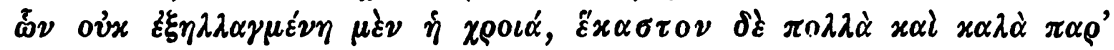

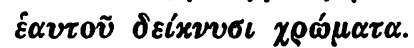

Wohl $\tilde{\varepsilon} x \alpha \sigma \tau o s$ (sc. $x i \omega \nu$ ).

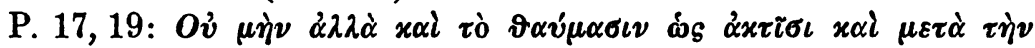

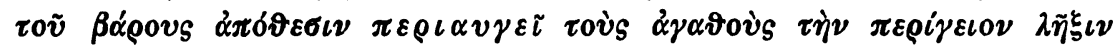

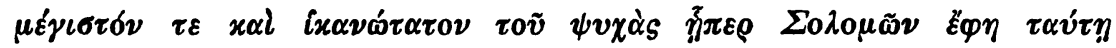

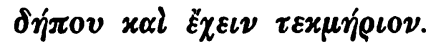

Der Sinn wird klar durch die Emendation $\pi \varepsilon \rho \iota \alpha v \gamma \varepsilon i \nu$.

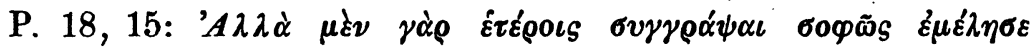

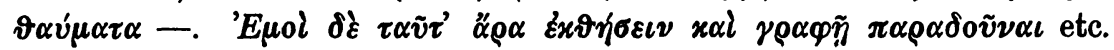

" $A \lambda \lambda \alpha \mu \dot{\nu} \nu$ jò $\varrho$ ?

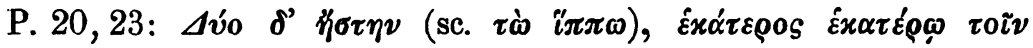

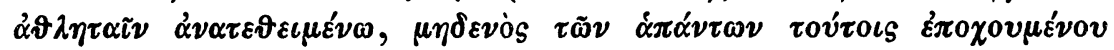

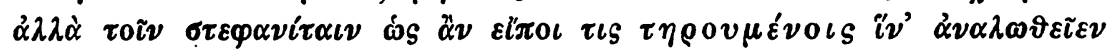

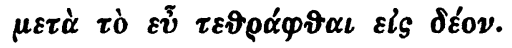

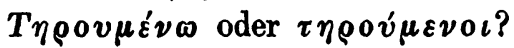




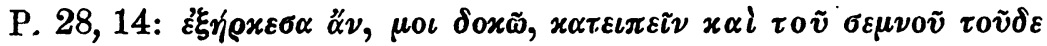

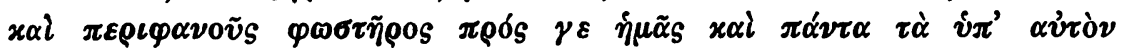
$\grave{\omega} \varphi \varepsilon \dot{\varepsilon} \lambda \iota \iota \boldsymbol{\nu}$.

$K \alpha \downarrow\langle\tau \eta \dot{\nu}\rangle \tau \sigma \tilde{v}$ und $\pi \rho \delta \delta s \varepsilon \dot{\eta} \mu \tilde{\alpha} s ?$

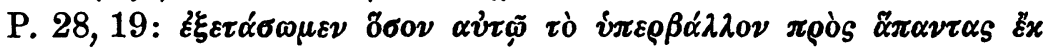

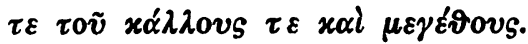

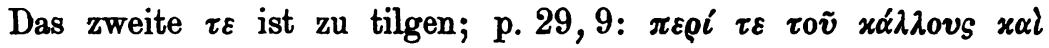
$\mu \varepsilon \gamma \varepsilon \dot{q} \vartheta$ ovs.

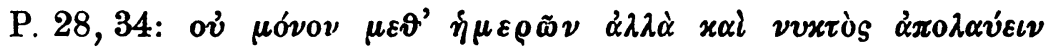

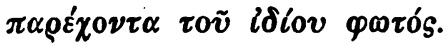

Schr. $\mu \varepsilon \vartheta^{\prime} \dot{\eta} \mu \varepsilon^{\prime} \rho \alpha \nu$.

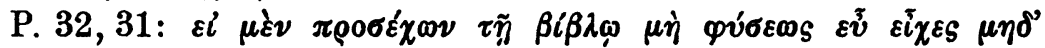

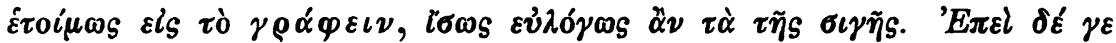

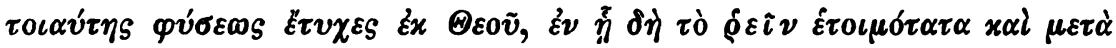

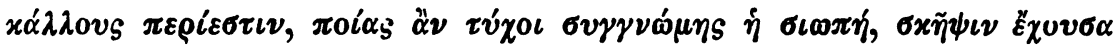

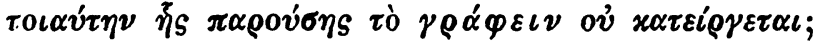

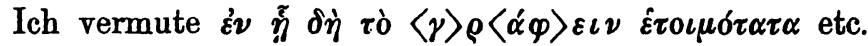

P. 37, 16:

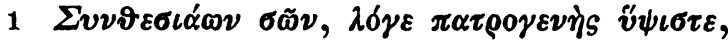

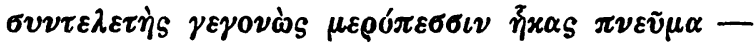

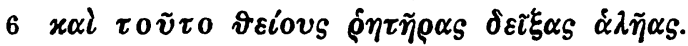

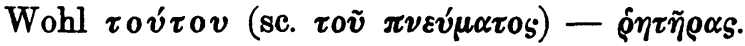

P. 38, 4:

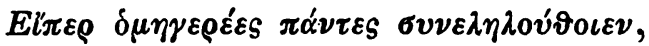

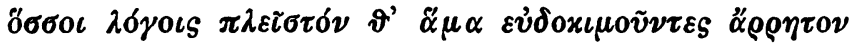

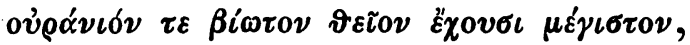

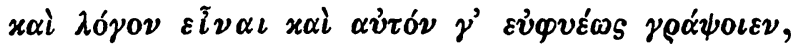

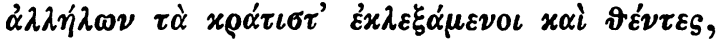

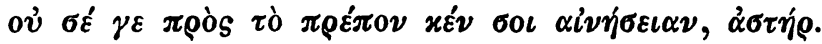

Viell. $\vartheta \alpha \mu \grave{\alpha}$ (st. $\vartheta^{\prime} \alpha_{\alpha}^{\prime} \mu \alpha$ ) und $\varepsilon i \tau \alpha$ st. $\varepsilon^{i} \nu \alpha \iota$ (die Hs $\varepsilon^{i} \nu \alpha$ ).

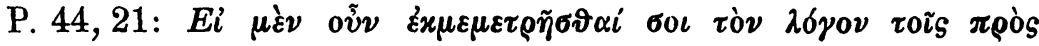

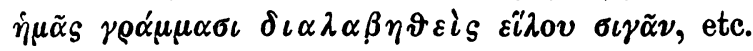

$\Delta \iota \varepsilon v \lambda \alpha \beta \eta \vartheta \varepsilon i s ?$

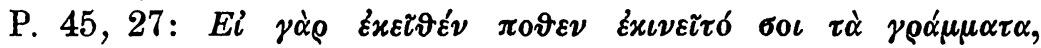

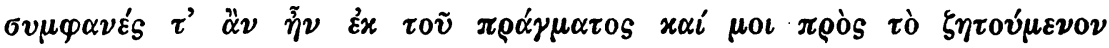

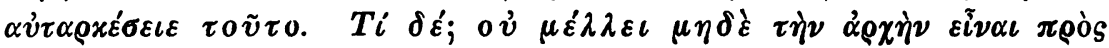
$\dot{\eta} \mu \check{\alpha} S$ бov $\varphi \iota \lambda i \alpha \nu \pi \iota \sigma \tau 0 v \dot{\mu} \mu \varepsilon 0 \nu$.

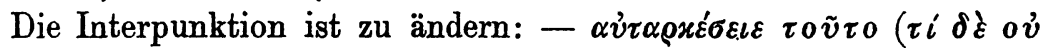
$\mu \varepsilon^{\prime} \lambda \lambda \varepsilon \iota$;) $\mu \eta \delta \dot{\varepsilon}-\pi \iota \sigma \tau 0 v^{\prime} \mu \varepsilon \nu 0 \nu$.

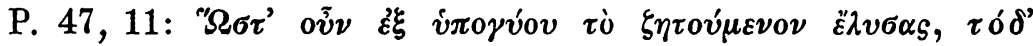

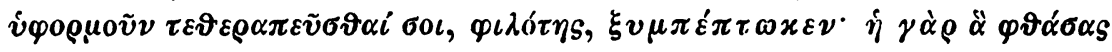




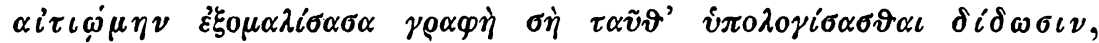

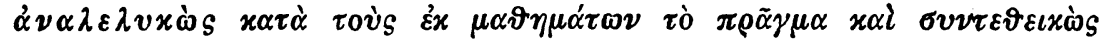

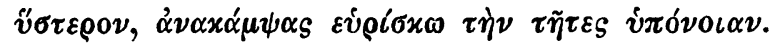

Die Hs bietet $\tau \sigma^{\prime} \vartheta^{\prime}$, d. i.: - $\varepsilon^{\prime} \lambda v \sigma \alpha S \tau \sigma^{\prime} \vartheta^{\prime} \dot{v} \varphi 0 \rho \mu o \tilde{v} \nu-\xi v \mu \pi \varepsilon^{\prime}-$

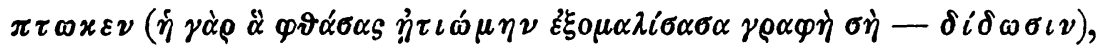
$\dot{\alpha} \nu \alpha \lambda \varepsilon \lambda v x \grave{\omega} s$ etc.

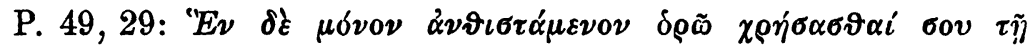

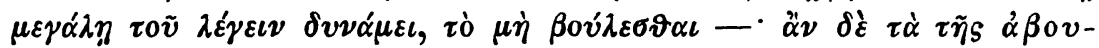
$\lambda i \alpha \varsigma x \rho \alpha \tau \tilde{\eta}$ etc.

$' A \beta 0 v \lambda\langle\eta \sigma\rangle i \alpha$ s?

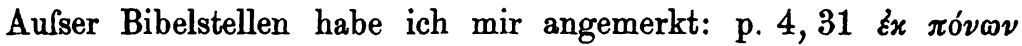

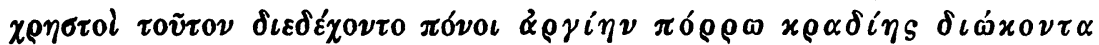

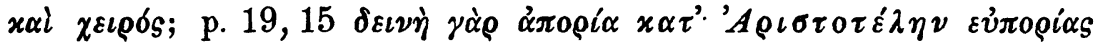

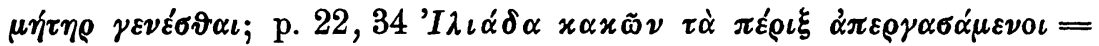
Zenob. IV 43 (Paroem. Gr. I p. 96, 17), Diogen. V 26 (P. G. I p. 256, 1)

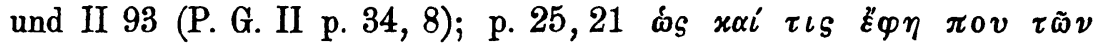

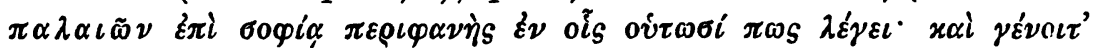

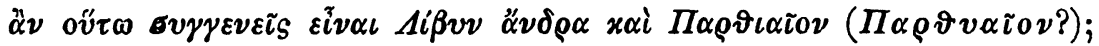

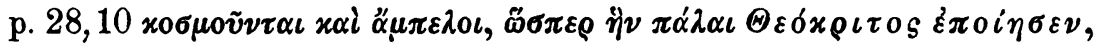

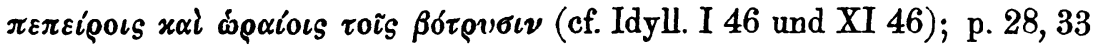

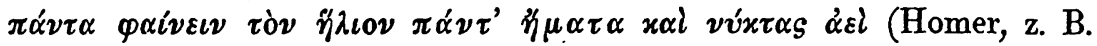

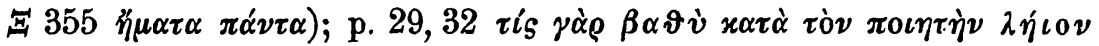

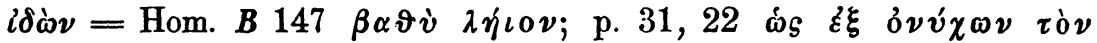
$\lambda \varepsilon^{\prime} 0 \nu \tau \alpha$, o̊ $\delta \dot{\eta} \varphi \alpha \sigma \iota=$ Diog. V 15 (P. G. I p. 252,1), Apost. VII 57

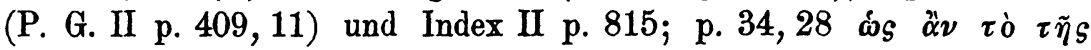

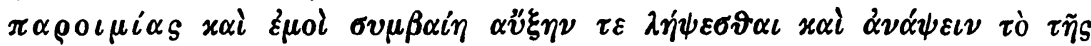

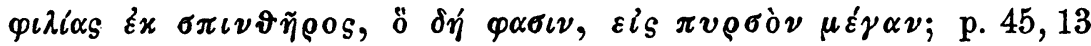

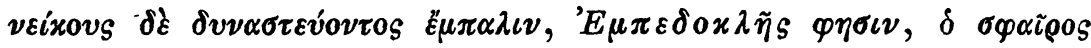

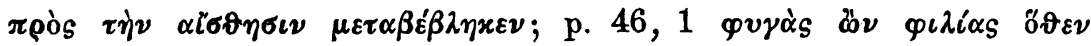

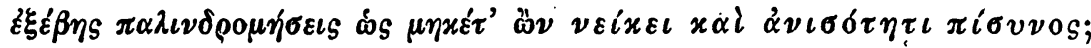

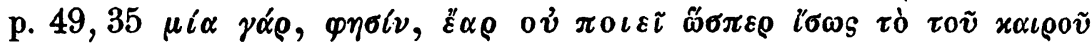

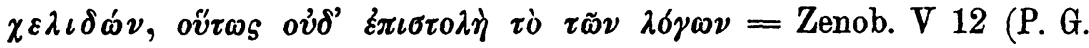
I 120,9).

Als neues Wort habe ich p. 45,8 $\pi \alpha \rho \alpha v \chi \varepsilon i \nu$ gefunden (wovon $\pi \alpha \rho \alpha \dot{v} \chi \eta \sigma \iota \varsigma$ bei Eustathios).

Thessalonike, 28. Nov. 1899.

P. N. Papageorgiu. 